\title{
WKB ANALYSIS OF GENERALIZED DERIVATIVE NONLINEAR SCHRÖDINGER EQUATIONS WITHOUT HYPERBOLICITY
}

\author{
RÉMI CARLES AND CLÉMENT GALLO
}

\begin{abstract}
We consider the semi-classical limit of nonlinear Schrödinger equations in the presence of both a polynomial nonlinearity and the derivative in space of a polynomial nonlinearity. By working in a class of analytic initial data, we do not have to assume any hyperbolic structure on the (limiting) phase/amplitude system. The solution, its approximation, and the error estimates are considered in time dependent analytic regularity.
\end{abstract}

\section{INTRODUCTION}

1.1. Setting. We consider the equation

(1.1) $i \varepsilon \partial_{t} u^{\varepsilon}+\frac{\varepsilon^{2}}{2} \partial_{x}^{2} u^{\varepsilon}+i \frac{\varepsilon}{2} \partial_{x}\left(g\left(\left|u^{\varepsilon}\right|^{2}\right) u^{\varepsilon}\right)-f\left(\left|u^{\varepsilon}\right|^{2}\right) u^{\varepsilon}=0, \quad(t, x) \in[0, T] \times \mathbb{R}$,

in the semi-classical limit $\varepsilon \rightarrow 0$, where $f, g$ are polynomials and $g(0)=f(0)=0$. The typical example we consider here is

$$
g(s)=\alpha s^{\gamma}, \quad f(s)=\lambda s^{\sigma}, \quad \text { where } \alpha, \lambda \in \mathbb{R} \text { and } \gamma, \sigma \in \mathbb{N} \backslash\{0\},
$$

but $f$ and $g$ need not be monomials. The initial data that we consider are WKB states (also known as Lagrangian states):

$$
u^{\varepsilon}(0, x)=a_{0}^{\varepsilon}(x) e^{i \phi_{0}^{\varepsilon}(x) / \varepsilon}=: u_{0}^{\varepsilon}(x),
$$

where $\phi_{0}^{\varepsilon}: \mathbb{R} \rightarrow \mathbb{R}$ is a real-valued phase, and $a_{0}^{\varepsilon}: \mathbb{R} \rightarrow \mathbb{C}$ is a possibly complexvalued amplitude. Our goal is to understand the semi-classical limit of equation (1.1), that is to describe the behaviour in the limit $\varepsilon \rightarrow 0$ of the solutions to (1.1) with initial data (1.3). We consider $\varepsilon$-dependent initial phase and amplitude, but they can be thought of as $\varepsilon$-independent, or having an asymptotic in powers of $\varepsilon$, as will be discussed below.

In the case $g=0$, we recover the more standard nonlinear Schrödinger equation, modelling for instance Bose-Einstein condensation (see e.g. [15, 23]). The case $f=0$ corresponds to the derivative Schrödinger equation, describing Alfvén waves (see e.g. [16, 19, 20, 25]). The cubic cases, $\alpha=0$ with $\sigma=1$, or $\gamma=\sigma=1$, with $\lambda=-1$, are known to be completely integrable (1, 27, 28, ). Derivation and analysis in non-cubic cases can be found in e.g. [10, 14, 17, 22.

This work was supported by the French ANR projects BECASIM (ANR-12-MONU-0007-04) and BoND (ANR-13-BS01-0009-01). 
1.2. Formal limit: hydrodynamical structure. Assuming that $a_{0}^{\varepsilon}$ is real-valued, the standard approach known as Madelung transform consists in seeking the solution $u^{\varepsilon}$ under the form $u^{\varepsilon}=a^{\varepsilon} e^{i \phi^{\varepsilon} / \varepsilon}$ with $a^{\varepsilon}$ and $\phi^{\varepsilon}$ real-valued. Plugging such an expression into (1.1) and separating real and imaginary parts yields

$$
\left\{\begin{array}{l}
\partial_{t} \phi^{\varepsilon}+\frac{1}{2}\left(\partial_{x} \phi^{\varepsilon}\right)^{2}+\frac{1}{2} g\left(\left|a^{\varepsilon}\right|^{2}\right) \partial_{x} \phi^{\varepsilon}+f\left(\left|a^{\varepsilon}\right|^{2}\right)=\frac{\varepsilon^{2}}{2} \frac{\partial_{x}^{2} a^{\varepsilon}}{a^{\varepsilon}}, \quad \phi_{\mid t=0}^{\varepsilon}=\phi_{0}^{\varepsilon}, \\
\partial_{t} a^{\varepsilon}+\partial_{x} \phi^{\varepsilon} \partial_{x} a^{\varepsilon}+\frac{1}{2} a^{\varepsilon} \partial_{x}^{2} \phi^{\varepsilon}+\frac{1}{2} \partial_{x}\left(g\left(\left|a^{\varepsilon}\right|^{2}\right) a^{\varepsilon}\right)=0, \quad a_{\mid t=0}^{\varepsilon}=a_{0}^{\varepsilon} .
\end{array}\right.
$$

Making this approach rigorous can be a delicate issue, especially when $a^{\varepsilon}$ has zeroes (see [5] and references therein). Remaining at a formal level, in the limit $\varepsilon \rightarrow 0$, the quantum pressure (right hand side of the equation for $\phi^{\varepsilon}$ ) vanishes, and we get

$$
\begin{cases}\partial_{t} \phi+\frac{1}{2}\left(\partial_{x} \phi\right)^{2}+\frac{1}{2} g\left(|a|^{2}\right) \partial_{x} \phi+f\left(|a|^{2}\right)=0, & \phi_{\mid t=0}=\phi_{0} \\ \partial_{t} a+\partial_{x} \phi \partial_{x} a+\frac{1}{2} a \partial_{x}^{2} \phi+\frac{1}{2} \partial_{x}\left(g\left(|a|^{2}\right) a\right)=0, & a_{\mid t=0}=a_{0},\end{cases}
$$

where we have supposed also that the initial phase and amplitude converge, $\phi_{0}^{\varepsilon} \rightarrow \phi_{0}$ and $a_{0}^{\varepsilon} \rightarrow a_{0}$, as $\varepsilon \rightarrow 0$. Note that this formal convergence remains in the case where $a_{0}^{\varepsilon}$ is complex-valued. Introducing $\rho=|a|^{2}$ and $v=\partial_{x} \phi$, (1.5) yields

$$
\left\{\begin{array}{l}
\partial_{t} v+v \partial_{x} v+\frac{1}{2} \partial_{x}(g(\rho) v)+\partial_{x} f(\rho)=0, \quad v_{\mid t=0}=\partial_{x} \phi_{0}, \\
\partial_{t} \rho+\partial_{x}(\rho v)+\partial_{x} Q(\rho)=0, \quad \rho_{\mid t=0}=\left|a_{0}\right|^{2}
\end{array}\right.
$$

where

$$
Q(\rho)=\rho g(\rho)-\frac{1}{2} \int_{0}^{\rho} g(r) d r .
$$

This is a generalized compressible Euler equation. We recover the standard isentropic Euler equation when $g=0$, with pressure law $p(\rho)=\rho f(\rho)-\int_{0}^{\rho} f(r) d r$.

1.3. Rigorous limit: mathematical setting. As noticed in [13, if $g=0$ and $f \in C^{\infty}$ is such that $f^{\prime}>0$ (not necessarily assuming that $f$ is polynomial), the system (1.6) is hyperbolic. Based on this important remark, it is possible to justify the semi-classical limit in Sobolev spaces $H^{s}(\mathbb{R}$ ), locally in time (so long as the solution to the Euler equation (1.6) remains smooth, that is, in particular, on a time interval independent of $\varepsilon$ ). The assumption $f^{\prime}>0$ was relaxed to cases where (1.6) is hyperbolic with $f^{\prime} \geqslant 0$ (the nonlinearity need not be cubic at the origin) in [2, 7.

The idea of Grenier consists in modifying the Madelung transform, by allowing the amplitude $a^{\varepsilon}$ to be complex-valued, and taking advantage to this new degree of freedom (compared to the Madelung transform) to consider

$$
\left\{\begin{array}{l}
\partial_{t} \phi^{\varepsilon}+\frac{1}{2}\left(\partial_{x} \phi^{\varepsilon}\right)^{2}+\frac{1}{2} g\left(\left|a^{\varepsilon}\right|^{2}\right) \partial_{x} \phi^{\varepsilon}+f\left(\left|a^{\varepsilon}\right|^{2}\right)=0, \quad \phi_{\mid t=0}^{\varepsilon}=\phi_{0}^{\varepsilon}, \\
\partial_{t} a^{\varepsilon}+\partial_{x} \phi^{\varepsilon} \partial_{x} a^{\varepsilon}+\frac{1}{2} a^{\varepsilon} \partial_{x}^{2} \phi^{\varepsilon}+\frac{1}{2} \partial_{x}\left(g\left(\left|a^{\varepsilon}\right|^{2}\right) a^{\varepsilon}\right)=\frac{i \varepsilon}{2} \partial_{x}^{2} a^{\varepsilon}, \quad a_{\mid t=0}^{\varepsilon}=a_{0}^{\varepsilon} .
\end{array}\right.
$$

We have written directly the system in the presence of $g$, in view of future references. It is readily checked that if $\left(\phi^{\varepsilon}, a^{\varepsilon}\right)$ solves (1.7), then $u^{\varepsilon}=a^{\varepsilon} e^{i \phi^{\varepsilon} / \varepsilon}$ solves (1.1). As suggested above, the good unknown to work in Sobolev spaces is not $\left(\phi^{\varepsilon}, a^{\varepsilon}\right)$, but rather $\left(\partial_{x} \phi^{\varepsilon}, a^{\varepsilon}\right)$, or even $\left(\partial_{x} \phi^{\varepsilon}, \operatorname{Re} a^{\varepsilon}, \operatorname{Im} a^{\varepsilon}\right)$. The system satisfied by this 
unknown (readily obtained from (1.7)) is a skew-symmetric perturbation of (the symmetric version of) (1.6).

In the case $g \neq 0$, the semi-classical limit for (1.1) was considered in 9] (case $f=0$ ) and [8] (with $f, g \in C^{\infty}\left(\mathbb{R}_{+} ; \mathbb{R}\right)$ ), by considering (1.7). However, in the case where $g \neq 0$, hyperbolicity is not a property that one has for free. In [9] (case $f=0$ ), the semi-classical analysis relies on the assumption

$$
\partial_{x} \phi^{\varepsilon} g^{\prime}>0
$$

where $\phi^{\varepsilon}$ appears in (1.7), and in [8], it relies on

$$
\partial_{x} \phi^{\varepsilon} g^{\prime}+f^{\prime}>0 \text {. }
$$

These assumptions are made to ensure the hyperbolicity for (1.7), but have the strong drawback to involve the solution itself.

To overcome this issue, we work in a functional setting where hyperbolicity is not needed. Assume $g=0$ : if $f^{\prime}<0(\lambda<0$ in (1.2) $)$, then the Euler equation (1.6) is elliptic. G. Métivier 18 has proved that in this case, the only reasonable $C^{1}$ solutions to (1.6) stem from analytic initial data. Indeed, if $\phi_{0}$ is analytic at some point $x_{0} \in \mathbb{R}$ and (1.6) has a $C^{1}$ solution, then $a_{0}$ is analytic at $x_{0}$. Therefore, if $\phi_{0}$ is analytic (e.g. $\left.\phi_{0}=0\right)$ and $a_{0}$ is not, then (1.6) has no $C^{1}$ solution. Conversely, if the initial data $a_{0}^{\varepsilon}$ and $\phi_{0}^{\varepsilon}$ are analytic, then the semi-classical limit for (1.1) with $g=0$ was studied in [11, 26], thanks to some tools developed by J. Sjöstrand [24, based on complex analysis. We shall also work with analytic regularity, but rather with a Fourier analysis point of view, introduced by J. Ginibre and G. Velo [12.

Following [12, for $w \geqslant 0$ and $\ell \geqslant 0$, we consider the space

$$
\mathcal{H}_{w}^{\ell}=\left\{\psi \in L^{2}(\mathbb{R}), \quad\|\psi\|_{\mathcal{H}_{w}^{\ell}}<\infty\right\}, \quad \text { where } \quad\|\psi\|_{\mathcal{H}_{w}^{\ell}}^{2}:=\int_{\mathbb{R}}\langle\xi\rangle^{2 \ell} e^{2 w\langle\xi\rangle}|\hat{\psi}(\xi)|^{2} d \xi,
$$

with $\langle\xi\rangle=\sqrt{1+\xi^{2}}$, and where the Fourier transform is defined by

$$
\hat{\psi}(\xi)=\mathcal{F} \psi(\xi)=\frac{1}{\sqrt{2 \pi}} \int_{\mathbb{R}} e^{-i x \xi} \psi(x) d x .
$$

Note that if $\ell \geqslant 0$ and $w>0, \mathcal{H}_{w}^{\ell}$ is continuously embedded in all Sobolev spaces $H^{s}$ for $s \in \mathbb{R}$. The interest of considering a time-dependent, decreasing, weight $w$ is that energy estimates become similar to parabolic estimates, since

$$
\frac{d}{d t}\|\psi\|_{\mathcal{H}_{w}^{\ell}}^{2}=2 \operatorname{Re}\left\langle\psi, \partial_{t} \psi\right\rangle_{\mathcal{H}_{w}^{\ell}}+2 \dot{w}\|\psi\|_{\mathcal{H}_{w}^{\ell+1 / 2}}^{2} .
$$

The last term may be understood as a gain of regularity $(\dot{w}<0)$. Mimicking our approach in [6] (where convergence results for a numerical scheme in the semiclassical limit are established in the case $g=0$ ), we will consider solutions to (1.7) where the phase and the complex-valued amplitude both live in such spaces, for a weight $w=w(t)=w_{0}-M t$, where $w_{0}>0$ and $M>0$ are fixed. Such spaces are also reminiscent of the framework considered in [21]. More precisely, for $T>0$, we will work in spaces such as

$$
C\left([0, T], \mathcal{H}_{w}^{\ell}\right)=\left\{\psi \mid \mathcal{F}^{-1}\left(e^{w(t)\langle\xi\rangle} \hat{\psi}\right) \in C\left([0, T], \mathcal{H}_{0}^{\ell}\right)=C\left([0, T], H^{\ell}\right)\right\},
$$

where $H^{\ell}=H^{\ell}(\mathbb{R})$ is the standard Sobolev space, or

$$
L^{2}\left([0, T], \mathcal{H}_{w}^{\ell}\right)=L_{T}^{2} \mathcal{H}_{w}^{\ell}=\left\{\psi \mid \int_{0}^{T}\|\psi(t)\|_{\mathcal{H}_{w(t)}^{\ell}}^{2} d t<\infty\right\} .
$$


Phases and amplitudes belong to spaces

$$
Y_{w, T}^{\ell}=C\left([0, T], \mathcal{H}_{w}^{\ell}\right) \cap L_{T}^{2} \mathcal{H}_{w}^{\ell+1 / 2},
$$

and the fact that phase and amplitude do not have exactly the same regularity shows up in the introduction of the space

$$
X_{w, T}^{\ell}=Y_{w, T}^{\ell+1} \times Y_{w, T}^{\ell}
$$

which is reminiscent of the fact that in the hyperbolic case, the good unknown is $\left(\partial_{x} \phi^{\varepsilon}, a^{\varepsilon}\right)$ rather than $\left(\phi^{\varepsilon}, a^{\varepsilon}\right)$. The space $X_{w, T}^{\ell}$ is endowed with the norm

$$
\|(\phi, a)\|_{X_{w, T}^{\ell}}=\|\phi \phi\|_{\ell+1, T}+\|a\|_{\ell, T}
$$

where

$$
\|\psi\|_{\ell, t}^{2}=\max \left(\sup _{0 \leqslant s \leqslant t}\|\psi(s)\|_{\mathcal{H}_{w(s)}^{\ell}}^{2}, 2 M \int_{0}^{t}\|\psi(s)\|_{\mathcal{H}_{w(s)}^{\ell+1 / 2}}^{2} d s\right) .
$$

1.4. Main results. Our first result states local well-posedness for (1.7) in this functional framework. To lighten our statements as well as the proofs, we shall assume that $f$ and $g$ are of the form (1.2), but linear combinations of such functions could be addressed as well, with heavier notations only.

Theorem 1.1. Let $w_{0}>0, \ell>1$ and $\left(\phi_{0}^{\varepsilon}, a_{0}^{\varepsilon}\right)_{\varepsilon \in[0,1]}$ be a bounded family in $\mathcal{H}_{w_{0}}^{\ell+1} \times$ $\mathcal{H}_{w_{0}}^{\ell}$. Then, provided $M=M(\ell)>0$ is chosen sufficiently large, for all $\varepsilon \in[0,1]$, there is a unique solution $\left(\phi^{\varepsilon}, a^{\varepsilon}\right) \in X_{w, T}^{\ell}$ to (1.7), where $w(t)=w_{0}-$ Mt and $T=T(\ell)<w_{0} / M$. Moreover, up to the choice of a possibly larger value for $M$ (and consequently a smaller one for $T$ ), we have the estimates

$$
\left\|\phi^{\varepsilon}\right\|_{\ell+1, T}^{2} \leqslant 4\left\|\phi_{0}^{\varepsilon}\right\|_{\mathcal{H}_{w_{0}}^{\ell+1}}^{2}+\left\|a_{0}^{\varepsilon}\right\|_{\mathcal{H}_{w_{0}}^{\ell}}^{4 \sigma}, \quad\left\|a^{\varepsilon}\right\|_{\ell, T}^{2} \leqslant 2\left\|a_{0}^{\varepsilon}\right\|_{\mathcal{H}_{w_{0}}^{\ell}}^{2} .
$$

Unlike in the framework of Sobolev spaces, we do not have tame estimates in $\mathcal{H}_{w}^{\ell}$. This is the reason why the existence time in the above result depends a priori on $\ell$. (In the Sobolev case, the existence time for hyperbolic systems in $H^{s}$ does not depend on $s>d / 2+1$, thanks to tame estimates.) It is natural to consider that the map $\ell \mapsto M(\ell)$ is increasing. In other words, $T$ in Theorem 1.1 is a decreasing function of $\ell$.

Our second result states the convergence of the phase and of the complex amplitude as $\varepsilon \rightarrow 0$.

Theorem 1.2. Let $w_{0}>0$, $\ell>1,\left(\phi_{0}, a_{0}\right) \in \mathcal{H}_{w_{0}}^{\ell+2} \times \mathcal{H}_{w_{0}}^{\ell+1}$ and $\left(\phi_{0}^{\varepsilon}, a_{0}^{\varepsilon}\right)_{\varepsilon \in(0,1]}$ bounded in $\mathcal{H}_{w_{0}}^{\ell+1} \times \mathcal{H}_{w_{0}}^{\ell}$ such that

$$
r_{0}^{\varepsilon}:=\left\|\phi_{0}^{\varepsilon}-\phi_{0}\right\|_{\mathcal{H}_{w_{0}}^{\ell+1}}+\left\|a_{0}^{\varepsilon}-a_{0}\right\|_{\mathcal{H}_{w_{0}}^{\ell}} \underset{\varepsilon \rightarrow 0}{\longrightarrow} 0 .
$$

Let $M=M(\ell+1)$ and $T=T(\ell+1)$, as defined as in Theorem 1.1. Then there is an $\varepsilon$-independent $C>0$ such that for all $\varepsilon \in(0,1]$,

$$
\left\|\phi^{\varepsilon}-\phi\right\|_{\ell+1, T}+\left\|a^{\varepsilon}-a\right\|_{\ell, T} \leqslant C\left(r_{0}^{\varepsilon}+\varepsilon\right),
$$

where $\left(\phi^{\varepsilon}, a^{\varepsilon}\right)$ denotes the solution to (1.7) and $(\phi, a)$ is the solution to (1.5).

The fact that in the above statement, $T(\ell+1)(<T(\ell))$ is considered, and not simply $T(\ell)$, is reminiscent of the fact that to prove error estimates in WKB expansions, one has to pay some price in terms of regularity, even in the linear case when working in Sobolev spaces (see e.g. [4, Chapter 1]). 
However, regarding convergence of the wave functions, the previous result is not sufficient. Indeed, as fast as $\phi_{0}^{\varepsilon}$ and $a_{0}^{\varepsilon}$ may converge as $\varepsilon \rightarrow 0$, Theorem 1.2 at most guarantees that $\phi^{\varepsilon}-\phi=\mathcal{O}(\varepsilon)$, which only ensures that $a^{\varepsilon} e^{i \phi^{\varepsilon} / \varepsilon}-a e^{i \phi / \varepsilon}=\mathcal{O}(1)$, due to the rapid oscillations. However, the above convergence result suffices to infer the convergence of quadratic observables:

Corollary 1.3. Under the assumptions of Theorem 1.2, the position and momentum densities converge:

$$
\left|u^{\varepsilon}\right|^{2} \underset{\varepsilon \rightarrow 0}{\longrightarrow}|a|^{2}, \quad \text { and } \quad \operatorname{Im}\left(\varepsilon \bar{u}^{\varepsilon} \partial_{x} u^{\varepsilon}\right) \underset{\varepsilon \rightarrow 0}{\longrightarrow}|a|^{2} \partial_{x} \phi, \quad \text { in } L^{\infty}\left([0, T] ; L^{1} \cap L^{\infty}(\mathbb{R})\right) .
$$

In order to get a good approximation of the wave function $a^{\varepsilon} e^{i \phi^{\varepsilon} / \varepsilon}$, we have to approximate $\phi^{\varepsilon}$ up to an error which is small compared to $\varepsilon$. It will be done by adding a corrective term to $(\phi, a)$. For this purpose, we consider the system obtained by linearizing (1.7) about $(\phi, a)$, solution to (1.5),

$$
\left\{\begin{aligned}
\partial_{t} \phi_{1} & +\left(\partial_{x} \phi+\frac{1}{2} g\left(|a|^{2}\right)\right) \partial_{x} \phi_{1} \\
& +\left(g^{\prime}\left(|a|^{2}\right) \partial_{x} \phi+2 f^{\prime}\left(|a|^{2}\right)\right) \operatorname{Re}\left(\bar{a} a_{1}\right)=0, \quad \phi_{1 \mid t=0}=\phi_{10}, \\
\partial_{t} a_{1}+ & \partial_{x} \phi \partial_{x} a_{1}+\frac{1}{2} a_{1} \partial_{x}^{2} \phi+\partial_{x} a \partial_{x} \phi_{1}+\frac{1}{2} a \partial_{x}^{2} \phi_{1}+\frac{1}{2} \partial_{x}\left(g\left(|a|^{2}\right) a_{1}\right) \\
& +\frac{1}{2} \partial_{x}\left(2 a g^{\prime}\left(|a|^{2}\right) \operatorname{Re}\left(\bar{a} a_{1}\right)\right)=\frac{i}{2} \partial_{x}^{2} a, \quad a_{1 \mid t=0}=a_{10} .
\end{aligned}\right.
$$

We refer to [4 for a discussion on the appearance of these correctors, and in particular regarding cases where they are trivial or not. Provided $\left(\phi_{0}, a_{0}\right) \in \mathcal{H}_{w_{0}}^{\ell+3} \times \mathcal{H}_{w_{0}}^{\ell+2}$ (which implies $(\phi, a) \in X_{w, T}^{\ell+2}$ according to Theorem 1.1) and $\left(\phi_{10}, a_{10}\right) \in \mathcal{H}_{w_{0}}^{\ell+2} \times$ $\mathcal{H}_{w_{0}}^{\ell+1}$, we will see that the solution to (1.10) belongs to $X_{w, T}^{\ell+1}$, and our final result is the following.

Theorem 1.4. Let $w_{0}>0, \ell>1,\left(\phi_{0}, a_{0}\right) \in \mathcal{H}_{w_{0}}^{\ell+3} \times \mathcal{H}_{w_{0}}^{\ell+2},\left(\phi_{10}, a_{10}\right) \in \mathcal{H}_{w_{0}}^{\ell+2} \times \mathcal{H}_{w_{0}}^{\ell+1}$ and $\left(\phi_{0}^{\varepsilon}, a_{0}^{\varepsilon}\right)_{\varepsilon \in(0,1]}$ bounded in $\mathcal{H}_{w_{0}}^{\ell+1} \times \mathcal{H}_{w_{0}}^{\ell}$ such that

$$
r_{1}^{\varepsilon}:=\left\|\phi_{0}^{\varepsilon}-\phi_{0}-\varepsilon \phi_{10}\right\|_{\mathcal{H}_{w_{0}}^{\ell+1}}+\left\|a_{0}^{\varepsilon}-a_{0}-\varepsilon a_{10}\right\|_{\mathcal{H}_{w_{0}}^{\ell}}=o(\varepsilon) \text { as } \varepsilon \rightarrow 0
$$

Then, for $M=M(\ell+2)$ and $T=T(\ell+2)$ as in Theorem 1.1, there is an $\varepsilon$ independent $C>0$ such that for all $\varepsilon \in(0,1]$,

$$
\left\|\phi^{\varepsilon}-\phi-\varepsilon \phi_{1}\right\|_{\ell+1, T}+\left\|a^{\varepsilon}-a-\varepsilon a_{1}\right\|_{\ell, T} \leqslant C\left(r_{1}^{\varepsilon}+\varepsilon^{2}\right),
$$

where $\left(\phi^{\varepsilon}, a^{\varepsilon}\right)$ denotes the solution to (1.7), $(\phi, a)$ is the solution to (1.5), and $\left(\phi_{1}, a_{1}\right)$ is the solution to (1.10). In particular,

$$
\left\|u^{\varepsilon}-a e^{i \phi_{1}} e^{i \phi / \varepsilon}\right\|_{L^{\infty}\left([0, T] ; L^{2} \cap L^{\infty}(\mathbb{R})\right)}=\mathcal{O}\left(\frac{r_{1}^{\varepsilon}}{\varepsilon}+\varepsilon\right) \underset{\varepsilon \rightarrow 0}{\longrightarrow} 0 .
$$




\section{WELL-POSEDNESS}

In this section, $\varepsilon \in[0,1]$ is fixed. Solutions to (1.7) are constructed as limits of the solutions of the iterative scheme

$$
\left\{\begin{array}{c}
\partial_{t} \phi_{j+1}^{\varepsilon}+\frac{1}{2} \partial_{x} \phi_{j}^{\varepsilon} \partial_{x} \phi_{j+1}^{\varepsilon}+\frac{1}{2} g\left(\left|a_{j}^{\varepsilon}\right|^{2}\right) \partial_{x} \phi_{j+1}^{\varepsilon}+f\left(\left|a_{j}^{\varepsilon}\right|^{2}\right)=0 \\
\phi_{j+1 \mid t=0}^{\varepsilon}=\phi_{0}^{\varepsilon}, \\
\partial_{t} a_{j+1}^{\varepsilon}+\partial_{x} \phi_{j}^{\varepsilon} \partial_{x} a_{j+1}^{\varepsilon}+\frac{1}{2} a_{j+1}^{\varepsilon} \partial_{x}^{2} \phi_{j}^{\varepsilon}+\frac{1}{2} \partial_{x}\left(g\left(\left|a_{j}^{\varepsilon}\right|^{2}\right)\right) a_{j+1}^{\varepsilon} \\
+\frac{1}{2} h\left(\left|a_{j}^{\varepsilon}\right|^{2}\right) \frac{a_{j}^{\varepsilon}}{a_{j+1}^{\varepsilon}} \partial_{x} a_{j}^{\varepsilon}=\frac{i \varepsilon}{2} \partial_{x}^{2} a_{j+1}^{\varepsilon}, \quad a_{j+1 \mid t=0}^{\varepsilon}=a_{0}^{\varepsilon},
\end{array}\right.
$$

where $h(s)=g(s) / s$. The scheme is initialized with time-independent $\left(\phi_{0}^{\varepsilon}, a_{0}^{\varepsilon}\right) \in$ $\mathcal{H}_{w_{0}}^{\ell+1} \times \mathcal{H}_{w_{0}}^{\ell} \subset X_{w, T}^{\ell}$ for any $T>0$.

The scheme is well-defined: if $\ell>1$, for a given $\left(\phi_{j}^{\varepsilon}, a_{j}^{\varepsilon}\right) \in X_{w, T}^{\ell}$, 2.1) defines $\left(\phi_{j+1}^{\varepsilon}, a_{j+1}^{\varepsilon}\right)$. Indeed, in the first equation, $\phi_{j+1}^{\varepsilon}$ solves a linear transport equation with smooth coefficients, and the second equation is equivalent through the relation $v_{j+1}^{\varepsilon}=a_{j+1}^{\varepsilon} e^{i \phi_{j}^{\varepsilon} / \varepsilon}$ to the linear Schrödinger equation

$$
\begin{aligned}
& i \varepsilon \partial_{t} v_{j+1}^{\varepsilon}+\frac{\varepsilon^{2}}{2} \partial_{x}^{2} v_{j+1}^{\varepsilon} \\
& \quad=-\left(\partial_{t} \phi_{j}^{\varepsilon}+\frac{1}{2}\left(\partial_{x} \phi_{j}^{\varepsilon}\right)^{2}+\frac{i \varepsilon}{2} \partial_{x}\left(g\left(\left|a_{j}^{\varepsilon}\right|^{2}\right)\right)+\frac{i \varepsilon}{2} h\left(\left|a_{j}^{\varepsilon}\right|^{2}\right) \overline{a_{j}^{\varepsilon}} \partial_{x} a_{j}^{\varepsilon}\right) v_{j+1}^{\varepsilon}
\end{aligned}
$$

with initial condition

$$
v_{j+1 \mid t=0}^{\varepsilon}=a_{0}^{\varepsilon} e^{i \phi_{0}^{\varepsilon} / \varepsilon} .
$$

This is a linear Schrödinger equation with a smooth and bounded external timedependent potential, for which the existence of an $L^{2}$-solution is granted, by perturbative arguments (the potential is complex-valued).

We recall the following lemma, which is proved in [12.

Lemma 2.1. Let $m \geqslant 0$ and $s>1 / 2$. Then, there is a constant $C>0$, independent of $w \geqslant 0$, such that for every $\psi_{1}, \psi_{2} \in \mathcal{H}_{w}^{\max (m, s)}$,

$$
\left\|\psi_{1} \psi_{2}\right\|_{\mathcal{H}_{w}^{m}} \leqslant C\left(\left\|\psi_{1}\right\|_{\mathcal{H}_{w}^{m}}\left\|\psi_{2}\right\|_{\mathcal{H}_{w}^{s}}+\left\|\psi_{1}\right\|_{\mathcal{H}_{w}^{s}}\left\|\psi_{2}\right\|_{\mathcal{H}_{w}^{m}}\right) .
$$

The following lemma is a toolbox for all the forthcoming analysis.

Lemma 2.2. Let $\ell>1$ and $T>0$. Let $(\phi, a) \in X_{w, T}^{\ell}, \tilde{a} \in Y_{w, T}^{\ell+1}$ and $(F, G) \in$ $L^{2}\left([0, T], \mathcal{H}_{w}^{\ell+1 / 2} \times \mathcal{H}_{w}^{\ell-1 / 2}\right)$ such that

$$
\begin{aligned}
\partial_{t} \phi & =F, & \phi(0) & \in \mathcal{H}_{w_{0}}^{\ell+1}, \\
\partial_{t} a & =G+i \theta_{1} \partial_{x}^{2} a+i \theta_{2} \partial_{x}^{2} \tilde{a}, & a(0) & \in \mathcal{H}_{w_{0}}^{\ell},
\end{aligned}
$$

where $\theta_{1}, \theta_{2} \in \mathbb{R}$. Then

$$
\begin{aligned}
& \|\phi\|_{\ell+1, T}^{2} \leqslant\|\phi(0)\|_{\mathcal{H}_{w_{0}}^{\ell+1}}^{2}+\frac{1}{M}\|\phi\|_{\ell+1, T} \sqrt{2 M}\|F\|_{L_{T}^{2} \mathcal{H}_{w}^{\ell+1 / 2}}, \\
& \|a\|_{\ell, T}^{2} \leqslant\|a(0)\|_{\mathcal{H}_{w_{0}}^{\ell}}^{2}+\frac{1}{M}\|a\|_{\ell, T} \sqrt{2 M}\|G\|_{L_{T}^{2} \mathcal{H}_{w}^{\ell-1 / 2}}+\frac{\left|\theta_{2}\right|}{2 M}\|a \mid\|_{\ell, T}\|\tilde{a}\|_{\ell+1, T} .
\end{aligned}
$$

Moreover, there exists $C>0$ (that depends only on $\ell$ ) such that 
- If $F=\partial_{x} \psi_{1} \partial_{x} \psi_{2}$ with $\psi_{1}, \psi_{2} \in Y_{\ell+1, T}$, then

$$
\sqrt{2 M}\|F\|_{L_{T}^{2} \mathcal{H}_{w}^{\ell+1 / 2}} \leqslant C\left\|\psi_{1}\right\|_{\ell+1, T}\left\|\psi_{2}\right\|_{\ell+1, T}
$$

- If $F=\left(\prod_{j=1}^{2 n} b_{j}\right) \partial_{x} \psi$ with $\psi \in Y_{\ell+1, T}$ and $b_{j} \in Y_{\ell, T}$ for all $j$, then

$$
\sqrt{2 M}\|F\|_{L_{T}^{2} \mathcal{H}_{w}^{\ell+1 / 2}} \leqslant C\left(\prod_{j=1}^{2 n}\left\|b_{j}\right\|_{\ell, T}\right)\|\psi\|_{\ell+1, T} .
$$

- If $F=\prod_{j=1}^{2 n} b_{j}$ with $b_{j} \in Y_{\ell, T}$ for all $j$, then

$$
\sqrt{2 M}\|F\|_{L_{T}^{2} \mathcal{H}_{w}^{\ell+1 / 2}} \leqslant C \prod_{j=1}^{2 n}\left\|b_{j}\right\|_{\ell, T} .
$$

- If $G=\partial_{x} \psi \partial_{x} b$ with $\psi \in Y_{\ell+1, T}$ and $b \in Y_{\ell, T}$, then

$$
\sqrt{2 M}\|G\|_{L_{T}^{2} \mathcal{H}_{w}^{\ell-1 / 2}} \leqslant C\|\psi\|\left\|_{\ell+1, T}\right\| b \|_{\ell, T} .
$$

- If $G=b \partial_{x}^{2} \psi$ with $\psi \in Y_{\ell+1, T}$ and $b \in Y_{\ell, T}$, then

$$
\sqrt{2 M}\|G\|_{L_{T}^{2} \mathcal{H}_{w}^{\ell-1 / 2}} \leqslant C\|\psi\|\left\|_{\ell+1, T}\right\| b \|_{\ell, T} .
$$

- If $G=\left(\prod_{j=1}^{2 n} b_{j}\right) \partial_{x} b$ with $b, b_{j} \in Y_{\ell, T}$ for all $j$, then

$$
\sqrt{2 M}\|G\|_{L_{T}^{2} \mathcal{H}_{w}^{\ell-1 / 2}} \leqslant C\left(\prod_{j=1}^{2 n}\left\|b_{j}\right\|_{\ell, T}\right)\|b\|_{\ell, T} .
$$

Remark 2.3. In the proof given below, the assumption $\ell>1$ is used only to establish the last three estimates on $G$. The rest of the proof only requires the assumption $\ell>1 / 2$. Actually, even the estimates on $G$ can be proved under the condition $\ell>1 / 2$, thanks to a refined version of Lemma 2.1 (see [ $[$ ]). However, since it is not useful in the sequel to sharpen this assumption, we choose to make the stronger assumption $\ell>1$ for the sake of conciseness.

Proof of Lemma [2.2. For fixed $t \in[0, T]$, (1.8) yields

$$
\frac{d}{d t}\|\phi\|_{\mathcal{H}_{w(t)}^{\ell+1}}^{2}+2 M\|\phi\|_{\mathcal{H}_{w(t)}^{\ell+3 / 2}}^{2}=2 \operatorname{Re}\langle\phi, F\rangle_{\mathcal{H}_{w(t)}^{\ell+1}} \leqslant 2\|\phi\|_{\mathcal{H}_{w(t)}^{\ell+3 / 2}}\|F\|_{\mathcal{H}_{w(t)}^{\ell+1 / 2}}
$$

By integration and Cauchy-Schwarz inequality in time, we get, for every $t \in[0, T]$,

$$
\|\phi(t)\|_{\mathcal{H}_{w(t)}^{\ell+1}}^{2}+2 M \int_{0}^{t}\|\phi(\tau)\|_{\mathcal{H}_{w(\tau)}^{\ell+3 / 2}}^{2} d \tau \leqslant\|\phi(0)\|_{\mathcal{H}_{w_{0}}^{\ell+1}}^{2}+2\|\phi\|_{L_{T}^{2} \mathcal{H}_{w}^{\ell+3 / 2}}\|F\|_{L_{T}^{2} \mathcal{H}_{w}^{\ell+1 / 2}},
$$

hence (2.5). The proof of (2.6) is similar, taking into account that

$$
\operatorname{Re}\left\langle i \theta_{1} \partial_{x}^{2} a, a\right\rangle_{\mathcal{H}_{w(t)}^{\ell}}=0
$$

since $\theta_{1} \in \mathbb{R}$, and that

$$
\left|\left\langle i \theta_{2} \partial_{x}^{2} \tilde{a}, a\right\rangle_{\mathcal{H}_{w(t)}^{\ell}}\right|=\left|\theta_{2}\right|\left|\int\langle\xi\rangle^{2 \ell} e^{2 w\langle\xi\rangle} \xi^{2} \mathcal{F}(\tilde{a}) \overline{\mathcal{F}(a)} d \xi\right| \leqslant\left|\theta_{2}\right|\|a\|_{\mathcal{H}_{w(t)}^{\ell+1 / 2}}\|\tilde{a}\|_{\mathcal{H}_{w(t)}^{\ell+3 / 2}}
$$


In order to prove (2.7), let us first use (2.2) with $m=\ell+1 / 2$ and $s=\ell$ : for every $t \in[0, T]$, we obtain

$$
\|F\|_{\mathcal{H}_{w(t)}^{\ell+1 / 2}}=\left\|\partial_{x} \psi_{1} \partial_{x} \psi_{2}\right\|_{\mathcal{H}_{w(t)}^{\ell+1 / 2}} \lesssim\left(\left\|\psi_{1}\right\|_{\mathcal{H}_{w(t)}^{\ell+3 / 2}}\left\|\psi_{2}\right\|_{\mathcal{H}_{w(t)}^{\ell+1}}+\left\|\psi_{1}\right\|_{\mathcal{H}_{w(t)}^{\ell+1}}\left\|\psi_{2}\right\|_{\mathcal{H}_{w(t)}^{\ell+3 / 2}}\right) .
$$

Taking the $L^{2}$ norm in time in the last estimate, we get

$$
\|F\|_{L_{T}^{2} \mathcal{H}_{w}^{\ell+1 / 2}} \lesssim\left(\left\|\psi_{1}\right\|_{L_{T}^{2} \mathcal{H}_{w}^{\ell+3 / 2}}\left\|\psi_{2}\right\|_{L_{T}^{\infty} \mathcal{H}_{w}^{\ell+1}}+\left\|\psi_{1}\right\|_{L_{T}^{\infty} \mathcal{H}_{w}^{\ell+1}}\left\|\psi_{2}\right\|_{L_{T}^{2} \mathcal{H}_{w}^{\ell+3 / 2}}\right),
$$

hence (2.7). The proofs of (2.8) and (2.9) are similar, thanks to multiple uses of (2.2) with $m=\ell+1 / 2$ and $s=\ell$. The proofs of (2.10), (2.11) and (2.12) are also similar, except that (2.2) is now applied with $m=s=\ell-1 / 2>1 / 2$.

Proof of Theorem 1.1. In view of the equation satisfied by $\phi_{j+1}^{\varepsilon}$ in (2.1), Lemma 2.2 yields

$$
\begin{aligned}
\left\|\phi_{j+1}^{\varepsilon}\right\|_{\ell+1, T}^{2} \leqslant & \left\|\phi_{0}^{\varepsilon}\right\|_{\mathcal{H}_{w_{0}}^{\ell+1}}^{2}+\frac{C}{M}\left\|\phi_{j+1}^{\varepsilon}\right\|_{\ell+1, T}^{2}\left\|\phi_{j}^{\varepsilon}\right\|_{\ell+1, T}+\frac{C}{M}\left\|\phi_{j+1}^{\varepsilon}\right\|\left\|_{\ell+1, T}\right\| a_{j}^{\varepsilon} \|_{\ell, T}^{2 \sigma} \\
& +\frac{C}{M}\left\|\phi_{j+1}^{\varepsilon}\right\|_{\ell+1, T}^{2}\left\|a_{j}^{\varepsilon}\right\|_{\ell, T}^{2 \gamma}
\end{aligned}
$$

As for $a_{j+1}^{\varepsilon}$, we obtain in a similar way

$$
\left\|a_{j+1}^{\varepsilon}\right\|_{\ell, T}^{2} \leqslant\left\|a_{0}^{\varepsilon}\right\|_{\mathcal{H}_{w_{0}}^{\ell}}^{2}+\frac{C}{M}\left\|a_{j+1}^{\varepsilon}\right\|_{\ell, T}^{2}\left\|\phi_{j}^{\varepsilon}\right\|_{\ell+1, T}+\frac{C}{M}\left\|a_{j+1}^{\varepsilon}\right\|_{\ell, T}^{2}\left\|a_{j}^{\varepsilon}\right\|_{\ell, T}^{2 \gamma} .
$$

Under the condition

$$
\frac{C}{M}\left\|\phi_{j}^{\varepsilon}\right\|_{\ell+1, T} \leqslant \frac{1}{4}, \quad \frac{C}{M}\left\|a_{j}^{\varepsilon}\right\|_{\ell, T}^{2 \gamma} \leqslant \frac{1}{4},
$$

we infer

$$
\frac{1}{4}\left\|\phi_{j+1}^{\varepsilon}\right\|_{\ell+1, T}^{2} \leqslant\left\|\phi_{0}^{\varepsilon}\right\|_{\mathcal{H}_{w_{0}}^{\ell+1}}^{2}+\frac{C^{2}}{M^{2}}\left\|a_{j}^{\varepsilon}\right\|_{\ell, T}^{4 \sigma}, \quad \frac{1}{2}\left\|a_{j+1}^{\varepsilon}\right\|_{\ell, T}^{2} \leqslant\left\|a_{0}^{\varepsilon}\right\|_{\mathcal{H}_{w_{0}}^{\ell}}^{2} .
$$

First step: boundedness of the sequence. We show by induction that, provided $M$ is sufficiently large, we can construct a sequence $\left(\phi_{j}^{\varepsilon}, a_{j}^{\varepsilon}\right)_{j \in \mathbb{N}}$ such that for every $j \in \mathbb{N}$,

$$
\left\|\phi_{j}^{\varepsilon}\right\|_{\ell+1, T}^{2} \leqslant 4\left\|\phi_{0}^{\varepsilon}\right\|_{\mathcal{H}_{w_{0}}^{\ell+1}}^{2}+\frac{4 C^{2}}{M^{2}}\left(2\left\|a_{0}^{\varepsilon}\right\|_{\mathcal{H}_{w_{0}}^{\ell}}^{2}\right)^{2 \sigma}, \quad\left\|a_{j}^{\varepsilon}\right\|_{\ell, T}^{2} \leqslant 2\left\|a_{0}^{\varepsilon}\right\|_{\mathcal{H}_{w_{0}}^{\ell}}^{2} .
$$

For that purpose, we choose $M$ sufficiently large such that (2.13) holds for $j=0$ and such that

$$
4\left\|\phi_{0}^{\varepsilon}\right\|_{\mathcal{H}_{w_{0}}^{\ell+1}}^{2}+\frac{4 C^{2}}{M^{2}}\left(2\left\|a_{0}^{\varepsilon}\right\|_{\mathcal{H}_{w_{0}}^{\ell}}^{2}\right)^{2 \sigma} \leqslant \frac{M^{2}}{16 C^{2}}, \quad\left(2\left\|a_{0}^{\varepsilon}\right\|_{\mathcal{H}_{w_{0}}^{\ell}}^{2}\right)^{\gamma} \leqslant \frac{M}{4 C} .
$$

Then, (2.15) holds for $j=0$, since with $\left(\phi_{0}^{\varepsilon}, a_{0}^{\varepsilon}\right)(t, x)=\left(\phi_{0}^{\varepsilon}, a_{0}^{\varepsilon}\right)(x)$ independent of time, it is easy to check that $\left\|\phi_{0}^{\varepsilon}\right\|_{\ell+1, T}=\left\|\phi_{0}^{\varepsilon}\right\|_{\mathcal{H}_{w_{0}}^{\ell+1}}$ and $\left\|a_{0}^{\varepsilon}\right\|_{\ell, T}=\left\|a_{0}^{\varepsilon}\right\|_{\mathcal{H}_{w_{0}}^{\ell}}$. Let $j \geqslant 0$ and assume that (2.15) holds. Then (2.15) and (2.16) ensure that the condition (2.13) is satisfied, and therefore (2.14) holds, from which we infer easily that (2.15) is true for $j$ replaced by $j+1$. 
Second step: convergence. For $j \geqslant 1$, we set $\delta \phi_{j}^{\varepsilon}=\phi_{j}^{\varepsilon}-\phi_{j-1}^{\varepsilon}$, and $\delta a_{j}^{\varepsilon}=$ $a_{j}^{\varepsilon}-a_{j-1}^{\varepsilon}$. Then, for every $j \geqslant 1$, we have

$$
\begin{aligned}
& \partial_{t} \delta \phi_{j+1}^{\varepsilon}+\frac{1}{2}\left(\partial_{x} \phi_{j}^{\varepsilon} \partial_{x} \delta \phi_{j+1}^{\varepsilon}+\partial_{x} \delta \phi_{j}^{\varepsilon} \partial_{x} \phi_{j}^{\varepsilon}\right)+\frac{1}{2} g\left(\left|a_{j}^{\varepsilon}\right|^{2}\right) \partial_{x} \delta \phi_{j+1}^{\varepsilon} \\
& \quad+\frac{1}{2}\left(g\left(\left|a_{j}^{\varepsilon}\right|^{2}\right)-g\left(\left|a_{j-1}^{\varepsilon}\right|^{2}\right)\right) \partial_{x} \phi_{j}^{\varepsilon}+f\left(\left|a_{j}^{\varepsilon}\right|^{2}\right)-f\left(\left|a_{j-1}^{\varepsilon}\right|^{2}\right)=0 .
\end{aligned}
$$

Taking into account that

$$
\left|a_{j}^{\varepsilon}\right|^{2 \gamma}-\left|a_{j-1}^{\varepsilon}\right|^{2 \gamma}=\sum_{k=0}^{\gamma-1}\left(a_{j-1}^{\varepsilon}\right)^{k} \delta a_{j}^{\varepsilon}\left(a_{j}^{\varepsilon}\right)^{\gamma-1-k}{\overline{a_{j}^{\varepsilon}}}^{\gamma}+\sum_{k=0}^{\gamma-1}\left(a_{j-1}^{\varepsilon}\right)^{\gamma}{\overline{a_{j-1}^{\varepsilon}}}^{k} \overline{\delta a_{j}^{\varepsilon} \bar{a}_{j}^{\varepsilon}}{ }^{\gamma-1-k},
$$

and that the same equality holds for $\gamma$ replaced by $\sigma$, Lemma 2.2 and (2.15) imply

$$
\left\|\delta \phi_{j+1}^{\varepsilon}\right\|_{\ell+1, T}^{2} \leqslant \frac{K}{M}\left(\left\|\delta \phi_{j+1}^{\varepsilon}\right\|_{\ell+1, T}^{2}+\left\|\delta \phi_{j}^{\varepsilon}\right\|_{\ell+1, T}^{2}+\left\|\delta a_{j}^{\varepsilon}\right\|_{\ell, T}^{2}\right)
$$

for some $K>0$, which does not depend on $\varepsilon$ provided $\left(\phi_{0}^{\varepsilon}, a_{0}^{\varepsilon}\right)_{\varepsilon \in[0,1]}$ is uniformly bounded in $\mathcal{H}_{w_{0}}^{\ell+1} \times \mathcal{H}_{w_{0}}^{\ell}$. Thus, for $M$ large enough,

$$
\left\|\delta \phi_{j+1}^{\varepsilon}\right\|_{\ell+1, T}^{2} \leqslant \frac{2 K}{M}\left(\left\|\delta \phi_{j}^{\varepsilon}\right\|_{\ell+1, T}^{2}+\left\|\delta a_{j}^{\varepsilon}\right\|_{\ell, T}^{2}\right) .
$$

Similarly, $\delta a_{j+1}^{\varepsilon}$ solves

$$
\begin{aligned}
\partial_{t} & \delta a_{j+1}^{\varepsilon}+\partial_{x} \phi_{j}^{\varepsilon} \partial_{x} \delta a_{j+1}^{\varepsilon}+\partial_{x} \delta \phi_{j}^{\varepsilon} \partial_{x} a_{j}^{\varepsilon}+\frac{1}{2} \delta a_{j+1}^{\varepsilon} \partial_{x}^{2} \phi_{j}^{\varepsilon}+\frac{1}{2} a_{j}^{\varepsilon} \partial_{x}^{2} \delta \phi_{j}^{\varepsilon} \\
& +\frac{1}{2} \partial_{x}\left(g\left(\left|a_{j}^{\varepsilon}\right|^{2}\right)\right) \delta a_{j+1}^{\varepsilon}+\frac{1}{2} \partial_{x}\left(g\left(\left|a_{j}^{\varepsilon}\right|^{2}\right)-g\left(\left|a_{j-1}^{\varepsilon}\right|^{2}\right)\right) a_{j}^{\varepsilon} \\
& +\frac{1}{2} h\left(\left|a_{j}^{\varepsilon}\right|^{2}\right) \partial_{x} a_{j}^{\varepsilon} \overline{a_{j}^{\varepsilon}} \delta a_{j+1}^{\varepsilon}+\frac{1}{2} h\left(\left|a_{j}^{\varepsilon}\right|^{2}\right) \partial_{x} a_{j}^{\varepsilon} \overline{\delta a_{j}^{\varepsilon}} a_{j}^{\varepsilon} \\
& +\frac{1}{2} h\left(\left|a_{j}^{\varepsilon}\right|^{2}\right) \partial_{x} \delta a_{j}^{\varepsilon} \overline{a_{j-1}^{\varepsilon}} a_{j}^{\varepsilon}+\frac{1}{2}\left(h\left(\left|a_{j}^{\varepsilon}\right|^{2}\right)-h\left(\left|a_{j-1}^{\varepsilon}\right|^{2}\right)\right) \partial_{x} a_{j-1}^{\varepsilon} \overline{a_{j-1}^{\varepsilon}} a_{j}^{\varepsilon} \\
& =i \frac{\varepsilon}{2} \partial_{x}^{2} \delta a_{j+1}^{\varepsilon},
\end{aligned}
$$

so Lemma 2.2 and (2.15) yield

$$
\left\|\delta a_{j+1}^{\varepsilon}\right\|_{\ell, T}^{2} \leqslant \frac{2 K}{M}\left(\left\|\delta \phi_{j}^{\varepsilon}\right\|_{\ell+1, T}^{2}+\left\|\delta a_{j}^{\varepsilon}\right\|_{\ell, T}^{2}\right) .
$$

We conclude that provided $\ell>1$, possibly increasing $M,\left(\phi_{j}^{\varepsilon}, a_{j}^{\varepsilon}\right)$ converges geometrically in $X_{w, T}^{\ell}$ as $j \rightarrow \infty$. Uniqueness of the solution $\left(\phi^{\varepsilon}, a^{\varepsilon}\right)$ to (1.7) follows from the same kind of estimates as the ones which prove the convergence.

\section{First ORDER APPROXIMATION}

Proof of Theorem 1.2. Next, assume that $\left(\phi_{0}, a_{0}\right) \in \mathcal{H}_{w_{0}}^{\ell+2} \times \mathcal{H}_{w_{0}}^{\ell+1}$. Then, in view of Theorem [1.1 the solution $(\phi, a)$ to (1.5) belongs to $X_{w, T}^{\ell+1}$. Given $\varepsilon>0$, if $\left(\phi_{0}^{\varepsilon}, a_{0}^{\varepsilon}\right) \in \mathcal{H}_{w_{0}}^{\ell+1} \times \mathcal{H}_{w_{0}}^{\ell}$, we denote by $\left(\phi^{\varepsilon}, a^{\varepsilon}\right)$ the solution to (1.7). We also denote $\left(\delta \phi^{\varepsilon}, \delta a^{\varepsilon}\right)=\left(\phi^{\varepsilon}-\phi, a^{\varepsilon}-a\right)$. Then, in the same fashion as above, we have

$$
\begin{aligned}
\partial_{t} \delta \phi^{\varepsilon} & +\frac{1}{2}\left(\partial_{x} \phi^{\varepsilon} \partial_{x} \delta \phi^{\varepsilon}+\partial_{x} \delta \phi^{\varepsilon} \partial_{x} \phi\right)+\frac{1}{2} g\left(\left|a^{\varepsilon}\right|^{2}\right) \partial_{x} \delta \phi^{\varepsilon} \\
& +\frac{1}{2}\left(g\left(\left|a^{\varepsilon}\right|^{2}\right)-g\left(|a|^{2}\right)\right) \partial_{x} \phi+f\left(\left|a^{\varepsilon}\right|^{2}\right)-f\left(|a|^{2}\right)=0,
\end{aligned}
$$


and

$$
\begin{aligned}
\partial_{t} & \delta a^{\varepsilon}+\partial_{x} \phi^{\varepsilon} \partial_{x} \delta a^{\varepsilon}+\partial_{x} \delta \phi^{\varepsilon} \partial_{x} a+\frac{1}{2} \delta a^{\varepsilon} \partial_{x}^{2} \phi^{\varepsilon}+\frac{1}{2} a \partial_{x}^{2} \delta \phi^{\varepsilon} \\
& +\frac{1}{2} \partial_{x}\left(g\left(\left|a^{\varepsilon}\right|^{2}\right)\right) \delta a^{\varepsilon}+\frac{1}{2} \partial_{x}\left(g\left(\left|a^{\varepsilon}\right|^{2}\right)-g\left(|a|^{2}\right)\right) a \\
& +\frac{1}{2} h\left(\left|a^{\varepsilon}\right|^{2}\right) \partial_{x} a^{\varepsilon} \overline{a^{\varepsilon}} \delta a^{\varepsilon}+\frac{1}{2} h\left(\left|a^{\varepsilon}\right|^{2}\right) \partial_{x} a^{\varepsilon} \overline{\delta a^{\varepsilon}} a \\
& +\frac{1}{2} h\left(\left|a^{\varepsilon}\right|^{2}\right) \partial_{x} \delta a^{\varepsilon}|a|^{2}+\frac{1}{2}\left(h\left(\left|a^{\varepsilon}\right|^{2}\right)-h\left(|a|^{2}\right)\right) \partial_{x} a|a|^{2}=i \frac{\varepsilon}{2} \partial_{x}^{2} \delta a^{\varepsilon}+i \frac{\varepsilon}{2} \partial_{x}^{2} a
\end{aligned}
$$

For some new constant $K$, Lemma 2.2 and Theorem 1.1 imply, for $M$ large enough,

$$
\left\|\delta \phi^{\varepsilon}\right\|_{\ell+1, T}^{2} \leqslant K\left\|\phi_{0}^{\varepsilon}-\phi_{0}\right\|_{\mathcal{H}_{w_{0}}^{\ell+1}}^{2}+\frac{K}{M}\left\|\delta a^{\varepsilon}\right\|_{\ell, T}^{2},
$$

and

$$
\left\|\delta a^{\varepsilon}\right\|_{\ell, T}^{2} \leqslant K\left\|a_{0}^{\varepsilon}-a_{0}\right\|_{\mathcal{H}_{w_{0}}^{\ell}}^{2}+\frac{K}{M}\left\|\delta \phi^{\varepsilon}\right\|_{\ell+1, T}^{2}+\frac{K}{M} \varepsilon\left\|\delta a^{\varepsilon}\right\|_{\ell, T}\|a\|_{\ell+1, T} .
$$

Possibly increasing the value of $M$ and adding the last two inequalities, we deduce

$$
\left\|\delta \phi^{\varepsilon}\right\|_{\ell+1, T}^{2}+\left\|\delta a^{\varepsilon}\right\|_{\ell, T}^{2} \leqslant C\left\|\phi_{0}^{\varepsilon}-\phi_{0}\right\|_{\mathcal{H}_{w_{0}}^{\ell+1}}^{2}+C\left\|a_{0}^{\varepsilon}-a_{0}\right\|_{\mathcal{H}_{w_{0}}^{\ell}}^{2}+C \varepsilon^{2},
$$

hence Theorem 1.2. As for the choice of $M$, a careful examination of the previous inequalities shows that aside from the assumption $M \geqslant M(\ell+1)$, which enables to estimate the source term, $M$ can be chosen as in Theorem 1.1 namely such that $M \geqslant M(\ell)$.

Proof of Corollary 1.3. Notice that, provided $w \geqslant 0$,

$$
\|\psi\|_{H^{\ell}(\mathbb{R})} \leqslant\|\psi\|_{\mathcal{H}_{w}^{\ell}} .
$$

In particular, Sobolev embedding yields, for $\ell>1 / 2$,

$$
\|\psi\|_{L^{\infty}(\mathbb{R})} \leqslant C\|\psi\|_{\mathcal{H}_{w}^{\ell}},
$$

where $C$ is independent of $w \geqslant 0$. With these remarks in mind, the $L^{1}$ estimates of Corollary 1.3 follow from Theorem 1.2 and Cauchy-Schwarz inequality, since

$$
\left\|\left|u^{\varepsilon}\right|^{2}-|a|^{2}\right\|_{L_{T}^{\infty} L^{1}}=\left\|\left|a^{\varepsilon}\right|^{2}-|a|^{2}\right\|_{L_{T}^{\infty} L^{1}} \leqslant\left\|a^{\varepsilon}+a\right\|_{L_{T}^{\infty} L^{2}}\left\|\delta a^{\varepsilon}\right\|_{L_{T}^{\infty} L^{2}},
$$

and

$$
\begin{aligned}
\left\|\operatorname{Im}\left(\varepsilon \bar{u}^{\varepsilon} \partial_{x} u^{\varepsilon}\right)-|a|^{2} \partial_{x} \phi\right\|_{L_{T}^{\infty} L^{1}} \leqslant & \varepsilon\left\|\operatorname{Im} \bar{a}^{\varepsilon} \partial_{x} a^{\varepsilon}\right\|_{L_{T}^{\infty} L^{1}}+\left\|\left|a^{\varepsilon}\right|^{2} \partial_{x} \phi^{\varepsilon}-|a|^{2} \partial_{x} \phi\right\|_{L_{T}^{\infty} L^{1}} \\
\leqslant & \varepsilon a^{\varepsilon}\left\|_{L_{T}^{\infty} H^{1}}^{2}+\right\| a^{\varepsilon}+a\left\|_{L_{T}^{\infty} L^{2}}\right\| \delta a^{\varepsilon}\left\|_{L_{T}^{\infty} L^{2}}\right\| \partial_{x} \phi \|_{L_{T}^{\infty} L^{\infty}} \\
& +\left\|a^{\varepsilon}\right\|_{L_{T}^{\infty} L^{\infty}}\left\|a^{\varepsilon}\right\|_{L_{T}^{\infty} L^{2}}\left\|\delta \phi^{\varepsilon}\right\|_{L_{T}^{\infty} H^{1}}
\end{aligned}
$$

The $L^{\infty}$ estimates in space follow by replacing $L^{1}$ and $L^{2}$ by $L^{\infty}$ in the above inequalities, and using Sobolev embedding again.

\section{Convergence of the Wave function}

Proof of Theorem 1.4. Let $\ell>1$, and $\left(\phi_{0}, a_{0}\right) \in \mathcal{H}_{w_{0}}^{\ell+2} \times \mathcal{H}_{w_{0}}^{\ell+1}$. Theorem 1.1 yields a unique solution $(\phi, a) \in X_{w, T}^{\ell+1}$ to (1.5). 
Let $\left(\phi_{10}, a_{10}\right) \in \mathcal{H}_{w_{0}}^{\ell+1} \times \mathcal{H}_{w_{0}}^{\ell}$. Like in Section 2] we note that (1.10) is a system of linear transport equations whose coefficients are smooth functions. The general theory of transport equations (see e.g. [3, Section 3]) then shows that (1.10) has a unique solution $\left(\phi_{1}, a_{1}\right) \in C\left([0, T], L^{2} \times L^{2}\right)$. We already know by this argument that the solution is actually more regular (in terms of Sobolev regularity), but we shall directly use a priori estimates in $\mathcal{H}_{w}^{\ell}$ spaces. Indeed, Lemma 2.2 implies that $\left(\phi_{1}, a_{1}\right) \in X_{w, T}^{\ell}$ with

$$
\begin{aligned}
& \left\|\phi_{1}\right\|_{\ell+1, T}^{2} \leqslant\left\|\phi_{10}\right\|_{\mathcal{H}_{w_{0}}^{\ell+1}}^{2}+\frac{C}{M}\left\|\phi_{1}\right\|_{\ell+1, T}^{2}\|\phi\|_{\ell+1, T}+\frac{C}{M}\left\|\phi_{1}\right\|_{\ell+1, T}^{2}\|a\|_{\ell, T}^{2 \gamma} \\
& \quad+\frac{C}{M}\left\|\phi_{1}\right\|\left\|_{\ell+1, T}\right\| \phi\left\|_{\ell+1, T}\right\| a\left\|_{\ell, T}^{2 \gamma-1}\right\| a_{1}\left\|_{\ell, T}+\frac{C}{M}\right\| \phi_{1}\left\|_{\ell+1, T}\right\| a\left\|_{\ell, T}^{2 \sigma-1}\right\| a_{1} \|_{\ell, T}
\end{aligned}
$$

along with

$$
\begin{gathered}
\left\|a_{1}\right\|_{\ell, T}^{2} \leqslant\left\|a_{10}\right\|_{\mathcal{H}_{w_{0}}^{\ell}}^{2}+\frac{C}{M}\left\|a_{1}\right\|_{\ell, T}\|a\|_{\ell, T}\left\|\phi_{1}\right\|_{\ell+1, T}+\frac{C}{M}\left\|a_{1}\right\|_{\ell, T}^{2}\|\phi\|_{\ell+1, T} \\
+\frac{C}{M}\left\|a_{1}\right\|_{\ell, T}^{2}\|a\|_{\ell, T}^{2 \gamma}+\frac{C}{M}\left\|a_{1}\right\|\left\|_{\ell, T}\right\| a \|_{\ell+1, T}
\end{gathered}
$$

for some $C>0$.

Let $\ell>1$. For $\left(\phi_{0}, a_{0}\right) \in \mathcal{H}_{w_{0}}^{\ell+3} \times \mathcal{H}_{w_{0}}^{\ell+2}$ and $\left(\phi_{10}, a_{10}\right) \in \mathcal{H}_{w_{0}}^{\ell+2} \times \mathcal{H}_{w_{0}}^{\ell+1}$, we consider:

- $(\phi, a) \in X_{w, T}^{\ell+2}$ the solution to (1.5).

- $\left(\phi_{1}, a_{1}\right) \in X_{w, T}^{\ell+1}$ the solution to (1.10).

- $\left(\phi_{\mathrm{app}}^{\varepsilon}, a_{\mathrm{app}}^{\varepsilon}\right)=(\phi, a)+\varepsilon\left(\phi_{1}, a_{1}\right)$.

- $\left(\phi^{\varepsilon}, a^{\varepsilon}\right) \in X_{w, T}^{\ell}$ the solution to (1.7).

We assume that $\left\|\phi_{0}^{\varepsilon}-\phi_{0}-\varepsilon \phi_{10}\right\|_{\mathcal{H}_{w_{0}}^{\ell+1}}=o(\varepsilon)$ and $\left\|a_{0}^{\varepsilon}-a_{0}-\varepsilon a_{10}\right\|_{\mathcal{H}_{w_{0}}^{\ell}}=o(\varepsilon)$. Set

$$
\begin{gathered}
\delta \phi_{1}^{\varepsilon}=\phi^{\varepsilon}-\phi_{\mathrm{app}}^{\varepsilon}=\phi^{\varepsilon}-\phi-\varepsilon \phi_{1}=\delta \phi^{\varepsilon}-\varepsilon \phi_{1}, \\
\delta a_{1}^{\varepsilon}=a^{\varepsilon}-a_{\mathrm{app}}^{\varepsilon}=a^{\varepsilon}-a-\varepsilon a_{1}=\delta a^{\varepsilon}-\varepsilon a_{1} .
\end{gathered}
$$

The equation satisfied by $\delta \phi_{1}^{\varepsilon}$ writes

$$
\begin{aligned}
& \partial_{t} \delta \phi_{1}^{\varepsilon}+\partial_{x} \phi \partial_{x} \delta \phi_{1}^{\varepsilon}+\frac{1}{2}\left|\partial_{x} \delta \phi^{\varepsilon}\right|^{2} \\
& +\frac{1}{2}\left(g\left(\left|a^{\varepsilon}\right|^{2}\right)-g\left(|a|^{2}\right)-2 g^{\prime}\left(|a|^{2}\right) \operatorname{Re}\left(\bar{a} \varepsilon a_{1}\right)\right) \partial_{x} \phi \\
& +\frac{1}{2}\left(g\left(\left|a^{\varepsilon}\right|^{2}\right)-g\left(|a|^{2}\right)\right) \varepsilon \partial_{x} \phi_{1}+\frac{1}{2} g\left(\left|a^{\varepsilon}\right|^{2}\right) \partial_{x} \delta \phi_{1}^{\varepsilon} \\
& +f\left(\left|a^{\varepsilon}\right|^{2}\right)-f\left(|a|^{2}\right)-2 f^{\prime}\left(|a|^{2}\right) \operatorname{Re}\left(\bar{a} \varepsilon a_{1}\right)=0
\end{aligned}
$$

Moreover, Taylor's formula yields

$$
\begin{aligned}
& g\left(\left|a^{\varepsilon}\right|^{2}\right)-g\left(|a|^{2}\right)-2 g^{\prime}\left(|a|^{2}\right) \operatorname{Re}\left(\bar{a} \varepsilon a_{1}\right) \\
& \quad=2 g^{\prime}\left(|a|^{2}\right) \operatorname{Re}\left(\bar{a} \delta a_{1}^{\varepsilon}\right)+4 \operatorname{Re}\left(\bar{a} \delta a^{\varepsilon}\right)^{2} \int_{0}^{1}(1-s) g^{\prime \prime}\left(\left|a+s \delta a^{\varepsilon}\right|^{2}\right) d s
\end{aligned}
$$

and the same identity holds for $g$ replaced by $f$. Thus, taking into account Theorem 1.1, which implies $\left\|\phi^{\varepsilon}\right\|_{\ell+1, T},\left\|a^{\varepsilon}\right\|_{\ell, T}=\mathcal{O}(1)$, and Theorem 1.2, which implies $\left\|\delta \phi^{\varepsilon}\right\|_{\ell+1, T},\left\|\delta a^{\varepsilon}\right\|_{\ell, T}=\mathcal{O}(\varepsilon)$, it follows from Lemma 2.2 that

$\left\|\delta \phi_{1}^{\varepsilon}\right\|_{\ell+1, T}^{2} \leqslant\left\|\phi_{0}^{\varepsilon}-\phi_{0}-\varepsilon \phi_{10}\right\|_{\mathcal{H}_{w_{0}}^{\ell}}^{2}+\frac{C}{M}\left\|\delta \phi_{1}^{\varepsilon}\right\|_{\ell+1, T}\left(\varepsilon^{2}+\left\|\delta \phi_{1}^{\varepsilon}\right\|_{\ell+1, T}+\left\|\delta a_{1}^{\varepsilon}\right\|_{\ell, T}\right)$. 
We deduce

$$
\left\|\delta \phi_{1}^{\varepsilon}\right\|_{\ell+1, T}^{2} \leqslant C\left\|\phi_{0}^{\varepsilon}-\phi_{0}-\varepsilon \phi_{10}\right\|_{\mathcal{H}_{w_{0}}^{\ell}}^{2}+\frac{C}{M} \varepsilon^{4}+\frac{C}{M}\left\|\delta a_{1}^{\varepsilon}\right\|_{\ell, T}^{2} .
$$

Similarly, $\delta a_{1}^{\varepsilon}$ solves

$$
\begin{aligned}
\partial_{t} & \delta a_{1}^{\varepsilon}+\partial_{x} \phi \partial_{x} \delta a_{1}^{\varepsilon}+\partial_{x} \delta \phi_{1}^{\varepsilon} \partial_{x} a+\partial_{x} \delta \phi^{\varepsilon} \partial_{x} \delta a^{\varepsilon} \\
& +\frac{1}{2} a \partial_{x}^{2} \delta \phi_{1}^{\varepsilon}+\frac{1}{2} \delta a_{1}^{\varepsilon} \partial_{x}^{2} \phi+\frac{1}{2} \delta a^{\varepsilon} \partial_{x}^{2} \delta \phi^{\varepsilon} \\
& +\partial_{x}\left[\left(g\left(\left|a^{\varepsilon}\right|^{2}\right)-g\left(|a|^{2}\right)-2 \varepsilon g^{\prime}\left(|a|^{2}\right) \operatorname{Re}\left(\bar{a} a_{1}\right)\right) a\right] \\
& +\partial_{x}\left[\left(g\left(\left|a^{\varepsilon}\right|^{2}\right)-g\left(|a|^{2}\right)\right) \varepsilon a_{1}\right]+\partial_{x}\left[g\left(\left|a^{\varepsilon}\right|^{2}\right) \delta a_{1}^{\varepsilon}\right]=\frac{i \varepsilon^{2}}{2} \partial_{x}^{2} a_{1}+\frac{i \varepsilon}{2} \partial_{x}^{2} \delta a_{1}^{\varepsilon} .
\end{aligned}
$$

From (4.1), Theorems 1.1 and 1.2, and Lemma 2.2, we deduce

$$
\left\|\delta a_{1}^{\varepsilon}\right\|_{\ell, T}^{2} \leq C\left\|a_{0}^{\varepsilon}-a_{0}-\varepsilon a_{10}\right\|_{\mathcal{H}_{w_{0}}^{\ell}}^{2}+\frac{C}{M} \varepsilon^{4}+\frac{C}{M}\left\|\delta \phi_{1}^{\varepsilon}\right\|_{\ell+1, T}^{2} .
$$

Adding (4.2) and (4.3), (1.11) follows. Like in the proof of Theorem 1.2 a careful examination of the inequalities that we have used shows that all the above estimates are valid provided that we assume $M \geqslant M(\ell)$, the constant provided by Theorem 1.1, and also $M \geqslant \max (M(\ell+1), M(\ell+2))$ in order to estimate the source terms.

To complete the proof of Theorem 1.4, consider the point-wise estimate

$$
\begin{aligned}
\left|a^{\varepsilon} e^{i \phi^{\varepsilon} / \varepsilon}-a e^{i \phi_{1}} e^{i \phi / \varepsilon}\right| & \leqslant\left|a^{\varepsilon}-a\right|+\left|a^{\varepsilon}\right|\left|e^{i \phi^{\varepsilon} / \varepsilon}-e^{i\left(\phi+\varepsilon \phi_{1}\right) / \varepsilon}\right| \\
& \leqslant\left|a^{\varepsilon}-a\right|+\left|a^{\varepsilon}\right|\left|2 \sin \left(\frac{\phi^{\varepsilon}-\phi-\varepsilon \phi_{1}}{2 \varepsilon}\right)\right| \\
& \leqslant\left|\delta a^{\varepsilon}\right|+\frac{1}{\varepsilon}\left|a^{\varepsilon}\right|\left|\delta \phi_{1}^{\varepsilon}\right| .
\end{aligned}
$$

We then conclude like in the proof of Corollary 1.3, by using Cauchy-Schwarz inequality, (3.1), and Sobolev embedding.

Remark 4.1. The last step in the above proof relies on the estimate $\delta \phi_{1}^{\varepsilon}=o(\varepsilon)$ in suitable spaces. Regarding the error estimate on $a^{\varepsilon}$, only $\delta a^{\varepsilon}$ appears. Recall however that $\left(\delta \phi_{1}^{\varepsilon}, \delta a_{1}^{\varepsilon}\right)$ solves a coupled system, so it is necessary to show that $\delta a_{1}^{\varepsilon}=o(\varepsilon)$ too (see also [4]).

Acknowledgements. The authors are grateful to Christof Sparber for attracting their attention on this problem.

\section{REFERENCES}

[1] M. J. Ablowitz and P. A. Clarkson, Solitons, nonlinear evolution equations and inverse scattering, vol. 149 of London Mathematical Society Lecture Note Series, Cambridge University Press, Cambridge, 1991.

[2] T. Alazard and R. Carles, Supercritical geometric optics for nonlinear Schrödinger equations, Arch. Ration. Mech. Anal., 194 (2009), pp. 315-347.

[3] H. Bahouri, J.-Y. Chemin, And R. DAnchin, Fourier analysis and nonlinear partial differential equations, vol. 343 of Grundlehren der Mathematischen Wissenschaften [Fundamental Principles of Mathematical Sciences], Springer, Heidelberg, 2011.

[4] R. CARLes, Semi-classical analysis for nonlinear Schrödinger equations, World Scientific Publishing Co. Pte. Ltd., Hackensack, NJ, 2008.

[5] R. Carles, R. Danchin, and J.-C. Saut, Madelung, Gross-Pitaevskii and Korteweg, Nonlinearity, 25 (2012), pp. 2843-2873. 
[6] R. Carles and C. Gallo, On Fourier time-splitting methods for nonlinear Schrödinger equations in the semi-classical limit II. Analytic regularity, Numer. Math. to appear. Archived at https://hal.archives-ouvertes.fr/hal-01271907.

[7] D. Chiron And F. Rousset, Geometric optics and boundary layers for nonlinear Schrödinger equations, Comm. Math. Phys., 288 (2009), pp. 503-546.

[8] B. Desjardins And C.-K. Lin, On the semiclassical limit of the general modified NLS equation, J. Math. Anal. Appl., 260 (2001), pp. 546-571.

[9] B. Desjardins, C.-K. Lin, And T.-C. Tso, Semiclassical limit of the derivative nonlinear Schrödinger equation, Math. Models Methods Appl. Sci., 10 (2000), pp. 261-285.

[10] É. Dumas, D. Lannes, And J. Szeftel, Variants of the focusing NLS equation: derivation, justification, and open problems related to filamentation, in Laser filamentation, CRM Ser. Math. Phys., Springer, Cham, 2016, pp. 19-75.

[11] P. GÉRARD, Remarques sur l'analyse semi-classique de l'équation de Schrödinger non linéaire, in Séminaire sur les Équations aux Dérivées Partielles, 1992-1993, École Polytech., Palaiseau, 1993, pp. Exp. No. XIII, 13. www.numdam.org

[12] J. Ginibre And G. Velo, Long range scattering and modified wave operators for some Hartree type equations. III. Gevrey spaces and low dimensions, J. Differential Equations, 175 (2001), pp. 415-501.

[13] E. Grenier, Semiclassical limit of the nonlinear Schrödinger equation in small time, Proc. Amer. Math. Soc., 126 (1998), pp. 523-530.

[14] N. HaYAShi AND T. OzAWA, Finite energy solutions of nonlinear Schrödinger equations of derivative type, SIAM J. Math. Anal., 25 (1994), pp. 1488-1503.

[15] C. Josserand and Y. Pomeau, Nonlinear aspects of the theory of Bose-Einstein condensates, Nonlinearity, 14 (2001), pp. R25-R62.

[16] M. Khanna AND R. RAJARAM, Evolution of nonlinear Alfvén waves propagating along the magnetic fields in a collisionless plasma, J. Plasma Phys., 28 (1982), pp. 459-468.

[17] X. Liu, G. Simpson, And C. Sulem, Stability of solitary waves for a generalized derivative nonlinear Schrödinger equation, J. Nonlinear Sci., 23 (2013), pp. 557-583.

[18] G. MÉTIVIER, Remarks on the well-posedness of the nonlinear Cauchy problem, in Geometric analysis of PDE and several complex variables, vol. 368 of Contemp. Math., Amer. Math. Soc., Providence, RI, 2005, pp. 337-356.

[19] K. Mio, T. Ogino, K. Minamy, and S. Takeda, Modified nonlinear Schrödinger equation for Alfvén waves propagating along the magnetic fiel in cold plasma, J. Phys. Soc. Japan, 41 (1976), pp. 265-273.

[20] E. MJøLHus, On the modulational instability of hydromagnetic waves parallel to the magnetic field, J. Plasma Phys., 16 (1976), pp. 321-334.

[21] C. Mounot And C. Villani, On Landau damping, Acta Math., 207 (2011), pp. 29-201.

[22] T. OzAWA, On the nonlinear Schrödinger equations of derivative type, Indiana Univ. Math. J., 45 (1996), pp. 137-163.

[23] L. Pitaevskit and S. Stringari, Bose-Einstein condensation, vol. 116 of International Series of Monographs on Physics, The Clarendon Press Oxford University Press, Oxford, 2003.

[24] J. SJöstrand, Singularités analytiques microlocales, in Astérisque, 95, vol. 95 of Astérisque, Soc. Math. France, Paris, 1982, pp. 1-166.

[25] C. Sulem And P.-L. Sulem, The nonlinear Schrödinger equation, Self-focusing and wave collapse, Springer-Verlag, New York, 1999.

[26] L. Thomann, Instabilities for supercritical Schrödinger equations in analytic manifolds, J. Differential Equations, 245 (2008), pp. 249-280.

[27] M. Wadati, K. Konno, and Y.-K. Ichikawa, A generalization of inverse scattering method, J. Phys. Soc. Japan, 46 (1979), pp. 1965-1966.

[28] V. E. Zakharov AND A. B. Shabat, Exact theory of two-dimensional self-focusing and onedimensional self-modulation of waves in nonlinear media, Ž. Èksper. Teoret. Fiz., 61 (1971), pp. 118-134.

CNRS \& Univ. Montpellier, Institut Montpelliérain Alexander Grothendieck, CC51,

Place E. Bataillon, 34095 Montpellier, France

E-mail address: Remi.Carles@math.cnrs.fr

E-mail address: Clement.Gallo@umontpellier.fr 